\title{
Fístula aortoesofágica após correção endovascular da dissecção de aorta torácica tipo B de Stanford
}

\author{
Aortic-esophageal fistula after endovascular repair of Stanford type B \\ thoracic aortic dissection
}

\author{
Cláudia Gurgel Marques ${ }^{1}$, Alexandre Fioranelli ${ }^{2}$, Gustavo Politzer Telles ${ }^{3}$, Paulo Saad ${ }^{3}$, Alvaro \\ Razuk Filho $^{4}$, Valter Khegam Karakhanian ${ }^{4}$, Valter Castelli Junior ${ }^{4}$, Roberto Augusto Caffaro ${ }^{5}$
}

\section{Resumo}

A correção endovascular da dissecção de aorta tipo B tem se mostrado como uma nova alternativa para reduzir o trauma cirúrgico. No entanto, as complicações de médio e longo prazo, tais como a fístula aortoesofágica, são ainda pouco conhecidas e pouco relatadas. O objetivo deste trabalho é descrever três casos de fístula aortoesofágica após o tratamento endovascular de 23 casos de dissecção de aorta descendente conduzidos pela equipe de Cirurgia Vascular da Santa Casa de São Paulo em um estudo retrospectivo. Esses pacientes apresentavam características em comum, como dissecção crônica, pós-operatório imediato sem intercorrências, necessidade de reintervenções, oclusão de troncos arteriais como a artéria subclávia, mesentérica, tronco celíaco, e, ainda, uma rápida evolução para o óbito após os primeiros sinais de fístula. Portanto, embora raramente descrita na literatura, a ocorrência de fístula aortoesofágica é uma complicação de causa até o momento indefinida do tratamento endovascular da dissecção de aorta descendente que merece atenção, dada sua recorrência e evolução fatal.

Palavras-chave: Dissecção, aorta, fístula, esôfago, complicações pós-operatórias.

\section{Introdução}

O tratamento endovascular da dissecção de aorta torácica tipo B de Stanford tem se mostrado, nos últimos anos, uma opção terapêutica para reduzir a mortalidade e morbidade precoces desses pacientes. No entanto, a evolução a médio e longo prazo ainda é pouco conhecida, uma vez que faltam, na literatura, estudos com grandes casuísticas descrevendo o seguimento pós-operatório desses pacientes ${ }^{1-3}$.

Essa nova alternativa terapêutica, associada ao tratamento clínico intensivo com controle rigoroso da pressão arterial, tem por objetivo ocluir o ponto de entrada proximal de modo a reduzir ou bloquear o fluxo para a falsa luz

\begin{abstract}
Endoluminal stent-graft for type B aortic dissection is a new alternative to reduce surgical trauma. However, medium- and long-term complications are still little known and poorly reported, such as the aortic-esophageal fistula. The objective of this study is to describe three cases of aortic-esophageal fistula after the endovascular treatment of 23 cases of descending aortic dissection conducted by the vascular surgery team of Santa Casa de São Paulo in a retrospective study. These patients presented some common characteristics: chronic dissection, successful early outcome, need of reinterventions, occlusion of arterial trunks such as subclavian artery, mesenteric artery, celiac trunk, and finally, a fast fatal course after the first fistula-related symptoms. Therefore, despite rarely described in the literature, aortic-esophageal fistula is a complication of the endovascular treatment of descending aortic dissection which demands attention due to its unpredictability, recurrence, and fatal outcome.
\end{abstract}

Keywords: Dissection, aorta, fistula, esophagus, postoperative complications.

de uma forma menos invasiva do que a da técnica cirúrgica aberta, sem a necessidade de toracotomia, clampeamento da aorta ou circulação extracorpórea ${ }^{4}$. A duração da cirurgia é menor, assim como as perdas sanguíneas e o tempo de internação ${ }^{4,5}$.

Por se tratar de uma técnica recente, os resultados em longo prazo ainda não estão bem definidos. Algumas complicações precoces, como surgimento de vazamentos ou endoleaks, acidentes vasculares cerebrais e isquemia de medula, já foram bem descritos na literatura ${ }^{6,7}$.

Já a fístula aortoesofágica é uma complicação até o momento pouco descrita. No entanto, o achado de três ca-

\footnotetext{
1. Cirurgiã vascular. Faculdade de Ciências Médicas da Santa Casa de São Paulo (FCMSCSP), São Paulo, SP.

2. Mestre em Cirurgia Vascular. Professor instrutor, FCMSCSP, São Paulo, SP.

3. Cirurgião vascular. Pós-graduando em Cirurgia Vascular, FCMSCSP, São Paulo, SP.

4. Doutor. Professor adjunto, disciplina de Cirurgia Vascular, FCMSCSP, São Paulo, SP.

5. Doutor. Professor e chefe da disciplina de Cirurgia Vascular, FCMSCSP, São Paulo, SP.

Não foram declarados conflitos de interesse associados à publicação deste artigo.

Artigo submetido em 06.04.09, aceito em 23.02.09.

J Vasc Bras. 2010;9(1):51-56.

Copyright $@ 2010$ by Sociedade Brasileira de Angiologia e de Cirurgia Vascular
} 
sos dessa complicação em um estudo prospectivo de 23 pacientes com dissecção de aorta tipo B tratados na Santa Casa de São Paulo, bem como a gravidade da sua evolução nos sugerem uma maior relevância desse tipo de intercorrência pós-operatória.

Neste estudo, descrevemos o surgimento de fístula aortoesofágica após tratamento endovascular da dissecção de aorta torácica descendente em três casos entre 23 pacientes tratados e relatamos a evolução deles, assim como as opções terapêuticas adotadas.

\section{Descrição dos casos}

Dos 23 pacientes tratados, três apresentaram fístula aortoesofágica no seguimento.

\section{Caso 1}

Paciente do sexo masculino, 70 anos, hipertenso e diabético, foi submetido ao tratamento endovascular devido à dilatação progressiva da dissecção de aorta torácica, atingindo diâmetro máximo da falsa luz de $50 \mathrm{~mm}$, diagnosticada nove meses antes. A dissecção se estendia desde a artéria subclávia esquerda até a ilíaca direita.

Utilizou-se uma prótese Gore $\mathrm{TAG}^{\circledR}$ (Gore, EUA), $37 \mathrm{~mm}$ de diâmetro e $20 \mathrm{~cm}$ de comprimento, que, embora tenha ocluído a origem da artéria subclávia esquerda, apresentava vazamento proximal ao final do procedimento sem reentrada distal.

Aos 11 meses de pós-operatório, porém, uma nova tomografia computadorizada (TC) revelou um aumento no diâmetro da falsa luz para $65 \mathrm{~mm}$, além do vazamento proximal na origem da artéria subclávia esquerda confirmado por angiografia. Optou-se pela embolização por acesso axilar esquerdo da origem da artéria subclávia com fio de alma móvel.

Nove meses depois, uma nova TC evidenciou a persistência do vazamento proximal. Dada a proximidade com a artéria carótida comum esquerda, o paciente foi submetido a derivação extra-anatômica carotídeo-carotídea cruzada, seguida de locação de uma nova endoprótese Gore TAG ${ }^{\circledR}$ proximal à primeira, ocluindo a origem da carótida comum esquerda.

O paciente se encontrava no $23^{\circ}$ mês pós-operatório da primeira cirurgia e, após 10 dias dessa última, apresentou hematêmese volumosa, evoluindo para óbito por choque hemorrágico. A necropsia identificou a presença de uma fístula aortoesofágica.

\section{Caso 2}

Paciente do sexo masculino, 52 anos, hipertenso e diabético, foi submetido ao tratamento endovascular devido ao aumento de $1 \mathrm{~cm}$ do diâmetro da falsa luz em 6 meses sob tratamento clínico. Apresentava dissecção de aorta torácica desde a artéria subclávia esquerda até as renais diagnosticada 19 meses antes.

Por ocasião do tratamento, a TC indicava uma falsa luz com diâmetro máximo de $70 \mathrm{~mm}$. A entrada proximal estava situada a $20 \mathrm{~mm}$ da artéria subclávia esquerda.

Foram utilizadas duas próteses Gore $\mathrm{TAG}^{\circledR}(40 \mathrm{~mm}$ de diâmetro e $15 \mathrm{~cm}$ de extensão), sem necessidade de oclusão da artéria subclávia. Ao final do procedimento, o paciente apresentava ponto de reentrada junto ao tronco celíaco, não havendo vazamento proximal.

Após 1 mês, o diâmetro da falsa luz havia aumentado para $89 \mathrm{~mm}$, e o vazamento persistia. Optou-se pela embolização da falsa luz com fio de alma móvel.

Dois meses depois, a tomografia indicou redução da falsa luz para $50 \mathrm{~mm}$, sem opacificação pela reentrada, e a presença de ar no esôfago.

O paciente foi então reinternado, apresentando fraqueza, dor lombar e febre. Evoluiu para óbito 3 dias depois em decorrência de uma hemorragia digestiva maciça. Durante o evento hemorrágico, a endoscopia digestiva evidenciou uma úlcera esofágica com sangramento ativo. A necropsia constatou uma fístula aortoesofágica.

\section{Caso 3}

Paciente de 57 anos, sexo masculino, hipertenso, com dissecção tipo B diagnosticada 15 meses antes, foi submetido ao tratamento devido à dilatação. A dissecção se estendia da artéria subclávia até as ilíacas, com diâmetro máximo da falsa luz de $55 \mathrm{~mm}$ na aorta torácica.

Optou-se por uma endoprótese Zenith ${ }^{\circledR}$ (Cook, EUA), $34 \mathrm{~mm}$ de diâmetro e $20 \mathrm{~cm}$ de extensão, com oclusão da artéria subclávia esquerda, que se encontrava a $12 \mathrm{~mm}$ da entrada proximal. Ao final do procedimento, não havia vazamento proximal, e observava-se um ponto de reentrada na origem do tronco celíaco. 
Após um pós-operatório precoce sem intercorrências, o paciente foi reinternado apresentando claudicação de membro superior esquerdo, dor torácica e queda hematimétrica no $15^{\circ}$ dia após a cirurgia, além de a TC indicar contraste na falsa luz, extravasamento de contraste em hemitórax esquerdo e persistência da reentrada no tronco celíaco (Figura 1). Esses achados foram confirmados por angiografia, além de um novo ponto de entrada na bifurcação das ilíacas. A conduta inicial foi locar um stent Palmaz $^{\circledR}$ (Cordis, EUA) na aorta, na altura do tronco celíaco e da artéria mesentérica superior, e, como o vazamento não cessou, optou-se pela injeção de cola (Hystoacril ${ }^{\circledR}$, Braun, Alemanha) na falsa luz, proximal a esse ponto (Figura 2).

Após uma evolução sem intercorrências por 14 dias, o paciente passou a apresentar dor torácica e febre, sendo di-

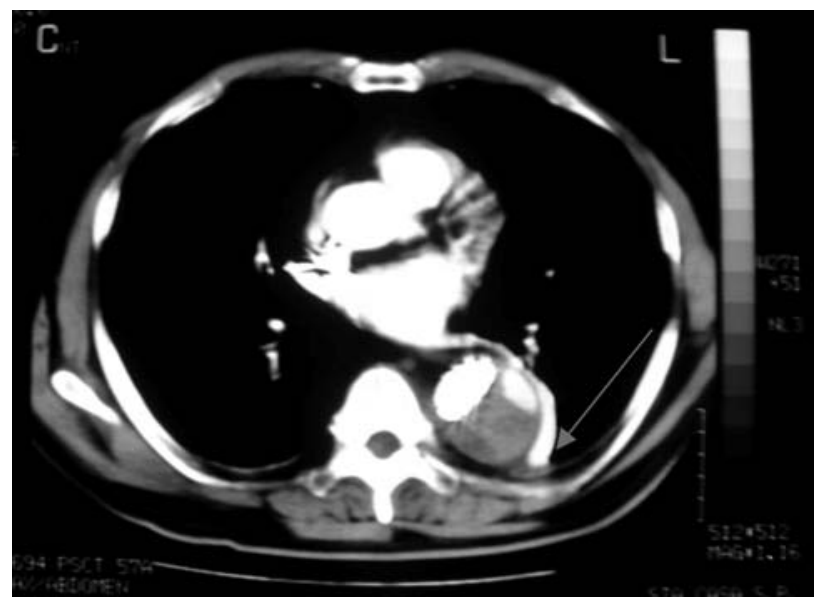

Figura 1 - Imagem em corte transversal de tomografia computadorizada após correção endovascular, notando-se a presença de endoprótese em luz verdadeira parcialmente colabada e persistência da luz falsa, com extravasamento de contraste para hemitórax esquerdo

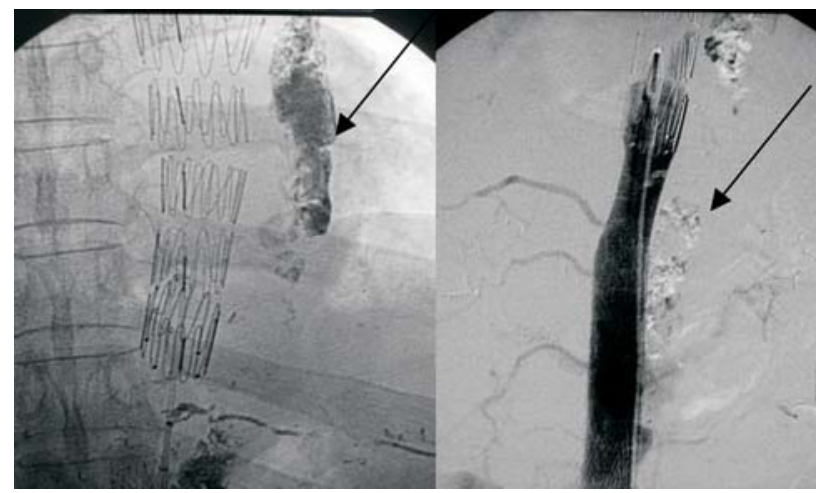

Figura 2 - Presença de cola em falsa luz, sem opacificação da mesma durante a arteriografia com subtração digital agnosticado um quadro de pneumonia hospitalar. Foi novamente internado e submetido a antibioticoterapia por um período total de 2 meses, recebendo vancomicina, polimixina e imipenem, sob terapia intensiva, com períodos de melhora clínica e de sepse. Três meses após a primeira cirurgia, uma cintilografia com leucócitos marcados evidenciou infecção do arco aórtico. Passou, então, a apresentar hematêmese, sendo submetido a uma endoscopia digestiva que detectou um abaulamento na parede do esôfago com um grande hematoma (Figura 3). Uma angiografia confirmou a hipótese de fístula aortoesofágica com origem no arco aórtico proximal à artéria subclávia esquerda (Figura 4).

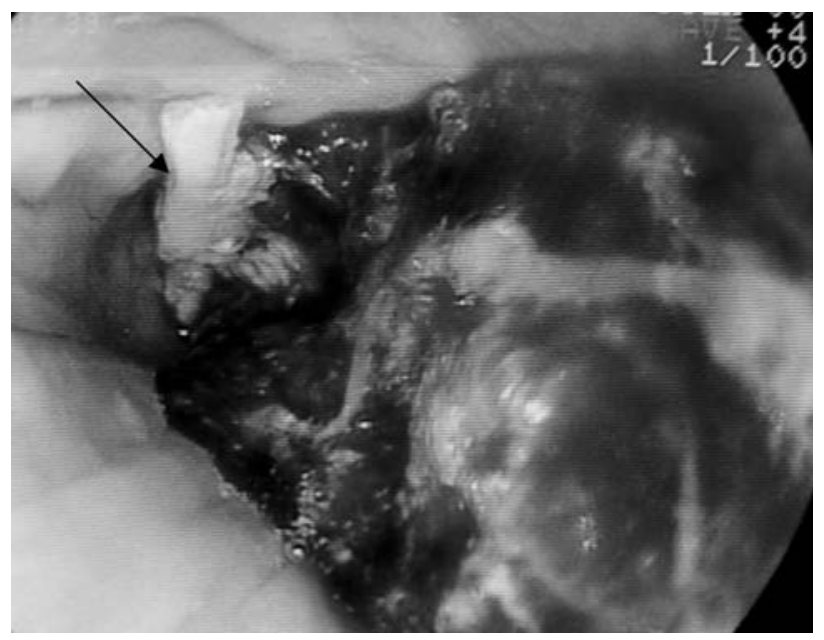

Figura 3 - Foto de endoscopia digestiva alta mostrando presença de restos alimentares e grande hematoma em parede esofágica sugerindo ponto de rotura da aorta torácica descendente

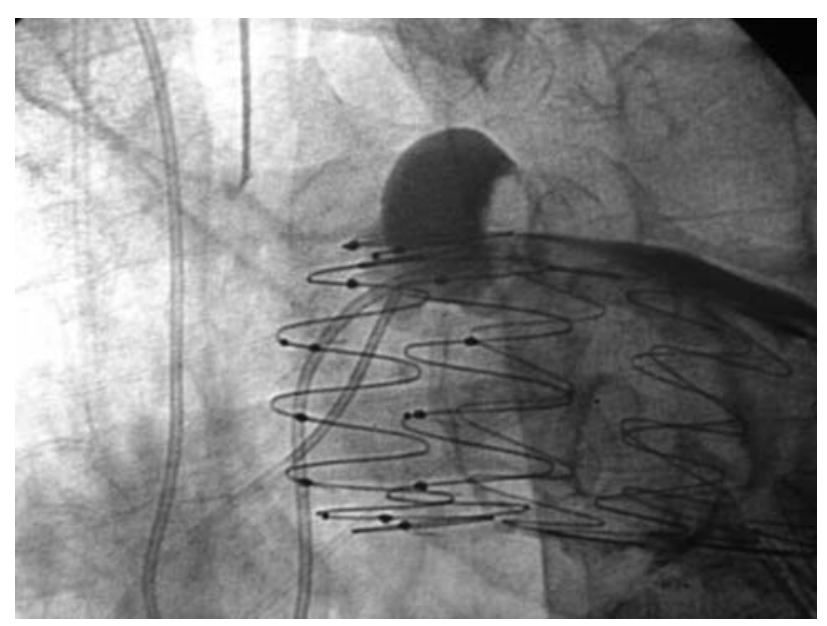

Figura 4 - Imagem arteriográfica por subtração digital identificando o ponto da fístula aortoesofágica 
Devido à proximidade com a artéria carótida comum esquerda, foi realizada uma derivação extra-anatômica carotídeo-carotídea cruzada, seguida da locação de uma nova endoprótese Zenith ${ }^{\circledR}$ (38 mm de diâmetro e $10 \mathrm{~cm}$ de extensão) proximal, a fim de ocluir a origem da fístula (Figura 5). Cinco dias após essa cirurgia, o paciente evoluiu para óbito por sepse e falência de múltiplos órgãos e sistemas. A fístula aortoesofágica foi identificada à necropsia e está apresentada na Figura 6.

A Tabela 1 mostra uma síntese da evolução dos três casos com suas características pré e pós-operatórias.

\section{Discussão}

O tratamento ideal da dissecção de aorta torácica Stanford B é ainda um tema controverso. A constatação da elevada mortalidade do tratamento cirúrgico aberto, de até $67 \%$ em algumas casuísticas, comparada aos $15 \%$ de mortalidade do tratamento clínico, levou, nos últimos anos, à restrição do tratamento cirúrgico aos casos que apresentassem complicações. No entanto, desde 1993, o tratamento endovascular vem se mostrando como uma nova alternativa para melhorar a evolução desses pacientes, por meio da oclusão do orifício de entrada proximal, de modo menos invasivo. Relatos de mortalidade hospitalar de 13\% têm até mesmo levado alguns centros de referência a adotar essa técnica sistematicamente ${ }^{4-6}$.

Por outro lado, os resultados animadores indicando redução da mortalidade precoce contrastam com a falta de

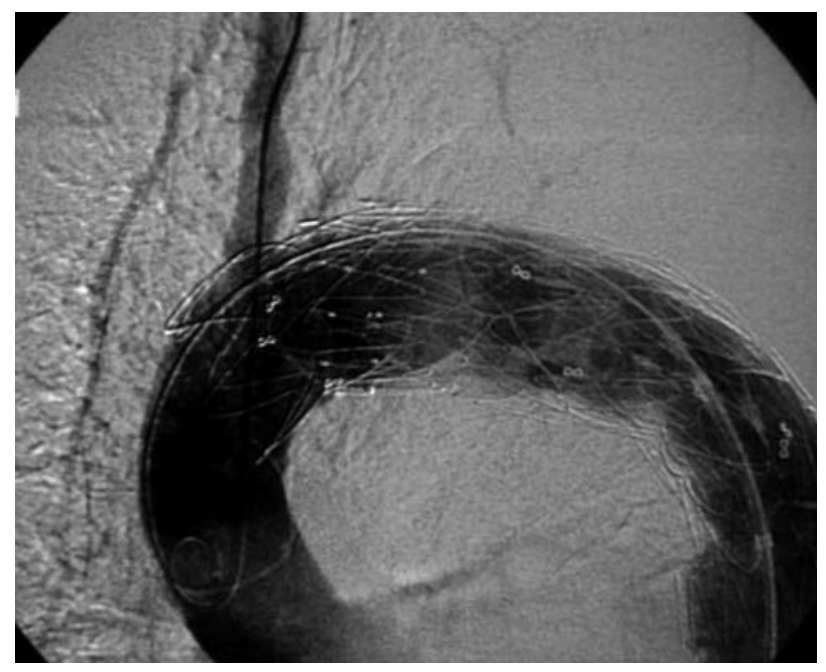

Figura 5 - Imagem de arteriografia por subtração digital em que se observa endoprótese proximal ao local da fístula aortoesofágica

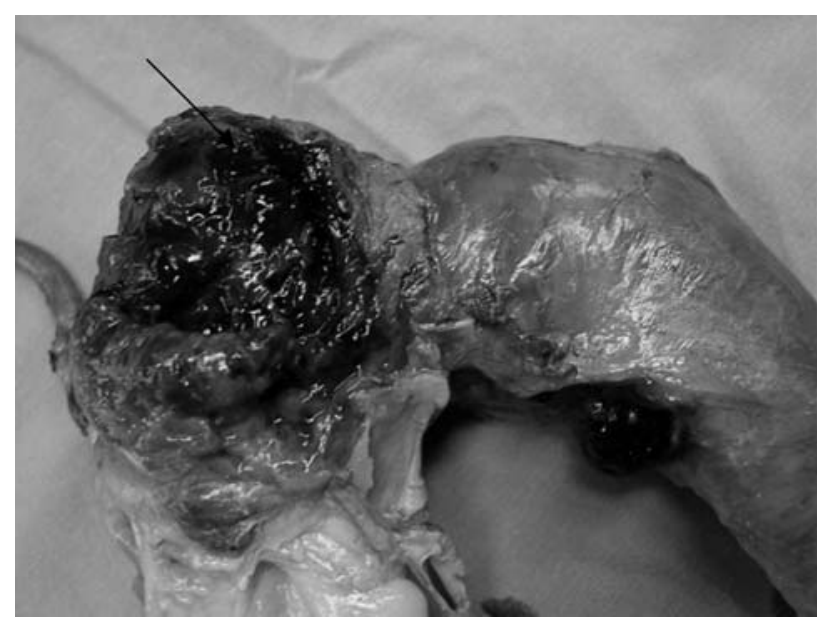

Figura 6 - Imagem fotográfica de peça de anatomia patológica de aorta torácica descendente em que notamos o ponto da fístula aortoesofágica

estudos a respeito da durabilidade ou da evolução tardia desses casos.

Nesta casuística de 23 pacientes com dissecção de aorta torácica descendente submetidos ao tratamento endovascular pela equipe de Cirurgia Vascular da Santa Casa de São Paulo, foram encontrados três casos de fístula aortoesofágica, complicação raramente descrita na literatura.

Alguns achados foram comuns aos três casos, como a cronicidade da dissecção, o tratamento indicado devido à dilatação da aorta, um pós-operatório imediato sem intercorrências, a presença de vazamento no seguimento de rotina, a necessidade de reintervenção antes mesmo do diagnóstico de fístula aortoesofágica (devido aos vazamentos), a necessidade de oclusão de ramos e troncos arteriais como o da subclávia, mesentérica superior e tronco celíaco e, finalmente, a evolução para óbito (Tabela 1).

De acordo com Porcu et al. ${ }^{6}$, o nível esofágico mais suscetível à formação de fístulas seria o seguimento distal ao brônquio esquerdo, onde a pobre vascularização poderia levar à necrose da parede do órgão, ao passo que nos terços proximal e distal do esôfago, a vascularização proveniente da artéria tireoidiana inferior, no primeiro, e diafragmática e gástrica esquerda, no último, atuariam de modo a proteger esses segmentos da isquemia. Embora a compressão direta pela endoprótese não possa ser descartada como causa dessas fístulas, a redução no diâmetro da aorta após o tratamento fala contra essa hipótese. 
Tabela 1 - Características clínicas dos casos de fístula aortoesofágica

\begin{tabular}{|c|c|c|c|}
\hline Características & Caso 1 & Caso 2 & Caso 3 \\
\hline Idade & 70 & 52 & 57 \\
\hline $\begin{array}{l}\text { Tempo de evolução entre o } \\
\text { diagnóstico e o tratamento } \\
\text { endovascular }\end{array}$ & 9 meses & 19 meses & 15 meses \\
\hline Indicação do tratamento & Dilatação & Dilatação & Dilatação \\
\hline $\begin{array}{l}\text { Diâmetro máximo da falsa luz } \\
\text { antes do tratamento } \\
\text { endovascular }\end{array}$ & $50 \mathrm{~mm}$ & $70 \mathrm{~mm}$ & $55 \mathrm{~mm}$ \\
\hline $\begin{array}{l}\text { Diâmetro máximo da falsa luz } \\
\text { no pós-operatório precoce }\end{array}$ & $45 \mathrm{~mm}$ & $89 \mathrm{~mm}$ & $48 \mathrm{~mm}$ \\
\hline Endoprótese & Gore $\mathrm{TAG}^{\circledR}-37-37-200$ & Gore $\mathrm{TAG}^{\circledR}-40-40-200$ & Zenith $^{\circledR}-34-34-200$ \\
\hline Oclusão arterial & Artéria subclávia esquerda & Artéria hepática & $\begin{array}{l}\text { Artéria subclávia } \\
\text { esquerda, tronco celíaco e } \\
\text { mesentérica superior }\end{array}$ \\
\hline $\begin{array}{l}\text { Reentrada distal após o } \\
\text { procedimento }\end{array}$ & Não & Sim & Sim \\
\hline Vazamento proximal & Sim & Não & Não \\
\hline Reintervenção & $\begin{array}{l}\text { 3: embolização da arteira } \\
\text { subclávia; bypass } \\
\text { carotídeo-carotídeo; endoprótese } \\
\text { proximal }\end{array}$ & $\begin{array}{l}\text { 1: embolização da falsa luz com } \\
\text { fio de alma móvel }\end{array}$ & $\begin{array}{l}\text { 3: stent + embolização da falsa } \\
\text { luz; bypass carotídeo-carotídeo; } \\
\text { endoprótese proximal }\end{array}$ \\
\hline $\begin{array}{l}\text { Tempo entre primeira interven- } \\
\text { ção e surgimento da fístula }\end{array}$ & 23 meses & 2 meses & 3 meses \\
\hline Óbito & $\operatorname{Sim}$ & $\operatorname{Sim}$ & $\operatorname{Sim}$ \\
\hline Causa imediata de óbito & Choque hemorrágico & Choque hemorrágico & Sepse \\
\hline $\begin{array}{l}\text { Confirmação do diagnóstico de } \\
\text { fístula aortoesofágica }\end{array}$ & Necropsia & Necropsia & Necropsia \\
\hline
\end{tabular}

Dos casos relatados, dois apresentaram redução da falsa luz após tratamento endovascular, e em ambos foi necessário ocluir a artéria subclávia esquerda, enquanto, no terceiro, a persistência de um ponto de reentrada e da expansão da aorta levaram à embolização da falsa luz e da artéria hepática comum. Neste último caso, a primeira tomografia após o tratamento evidenciou uma falsa luz de $89 \mathrm{~mm}$, ou seja, $19 \mathrm{~mm}$ maior do que no pré-operatório.

A hipótese da compressão direta da aorta sobre o esôfago foi defendida por Herolkl et al. ${ }^{7}$, quando relataram um caso de fístula entre 18 pacientes submetidos a esse tratamento por dissecção num paciente em quem a aorta apresentava $13 \mathrm{~cm}$ de diâmetro. A rotura da falsa luz no esôfago levou ao óbito no $62^{\circ}$ dia do pós-operatório.

Para Blockler et al. ${ }^{5}$, a rigidez dos sistemas endovasculares pode levar à penetração ou lesão da parede da aorta, sendo que a endoprótese ideal para dissecções deveria ser mais flexível e ainda não foi desenvolvida. Este autor também descreveu um caso fatal de fístula (porém aortobrônquica e não esofágica), à que atribuiu a compressão extrínseca, entre 37 casos de dissecção de aorta descendente submetidos ao tratamento endovascular. Dezessete meses após a cirurgia, esse paciente apresentou hemoptise maciça, tratada por meio da embolização da falsa luz com mola, evoluindo para óbito 2 semanas depois, quando apresentou outro episódio hemorrágico.

Já na casuística de Eggebrecht et al. ${ }^{4}$, dos 38 pacientes com dissecção de aorta tratados com endoprótese, um evoluiu com uma fístula aortoesofágica no pós-operatório tardio. A causa da complicação não chega a ser discutida no relato, e a hemorragia subsequente levou o paciente ao óbito.

\section{Conclusão}

Alguns achados são comuns a todos os casos descritos, como o início dos sintomas num seguimento tardio, 
após um pós-operatório sem intercorrências, e o curso fatal a despeito das diversas intervenções adotadas. Algumas hipóteses foram formuladas para explicar essa complicação, mas não há casuística na literatura suficiente para estabelecer os fatores de risco para seu surgimento. Embora imprevisível até o momento, parece-nos estar relacionada aos casos de aortas muito dilatadas ou àqueles em que há necessidade de oclusão da artéria subclávia esquerda ou de troncos viscerais, sendo recomendável sua revascularização extra-anatômica prévia sempre que factível. Sobretudo nesses casos, a suspeita clínica deverá ser conduzida com métodos diagnósticos invasivos como endoscopia, tomografia e angiografia precocemente a fim de não retardar o tratamento.

Com o aumento da experiência no tratamento endovascular da dissecção de aorta tipo B, o espectro das complicações tardias tem-se ampliado, incluindo eventos graves e letais, que demandam pesquisa detalhada e específica. Embora raramente descrita na literatura, a fistula aortoesofágica consiste numa complicação desastrosa, que levou todos os casos já relatados ao óbito. Como foi dito anteriormente, é de fundamental importância, neste momento, o desenvolvimento de estudos de seguimento em longo prazo do tratamento endovascular da dissecção de aorta torácica descendente capazes de responder a vários questionamentos e, certamente, de descrever essa e várias outras complicações raramente relatadas.

\section{Referências}

1. Goksel O, Tireli E, Kalko Y, et al. Mid-term outcome with surgery for type B aortic dissections: a single center experience. J Card Surg. 2006;23:27-30.
2. Kiyotaka I, Shinichi S, Keiji U. Early and medium-term results of stent-graft treatment of DeBakey type III aortic dissection. J Cardiovasc Surg. 2006;47:651-7.

3. Hutschala D, Fleck T, Czerny M, et al. Endoluminal stentgraft placement in patients with acute aortic dissection type B. Eur J Cardio-thorac Surg. 2002;21:964-9.

4. Eggebrecht H, Herold U, Kuhnt O, et al. Endovascular stentgraft treatment of aortic dissection: determinants of postinterventional outcome. Eur Heart J. 2005;26:489-97.

5. Blockler D, Schumacher H, Ganten M, et al. Complications after endovascular repair of acute symptomatic and chronic expanding Stanford type B aortic dissections. J Thorac Cardiovasc Surg. 2006;32:361-8.

6. Porcu P, Chvanon O, Sessa C, Thony F, Aubert A, Blin D. Esophageal fistula after endovascular treatment in a type B aortic dissection of the descending thoracic aorta. J Vasc Surg. 2005;41:708-11.

7. Herolkl U, Piotrowski J, Baumgart D, Eggebrecht H, Erbel R, Jakob H. Endoluminal stent graft repair for acute and chronic type B aortic dissection and atherosclerotic aneurysm of the thoracic aorta: an interdisciplinary task. Eur J Cardio-thorac Surg. 2002;22:891-7.

Correspondência:

Claudia Gurgel Marques

R. Maria Borba, 67/24, Vila Buarque

CEP 01221-040 - São Paulo, SP

E-mail: claudiagurgel@yahoo.com.br

\section{Contribuições dos autores}

Concepção e desenho do estudo: CGM, AF, AR Filho

Análise e interpretação dos dados: AF, VKK, VC Junior, RAC

Coleta de dados: CGM, GPT, PS

Redação do artigo: CGM, AF

Revisão crítica do texto: AF, VKK, VC Junior

Aprovação final do artigo*: CGM, AF, GPT, PS, AR Filho, VKK, VC Junior, RAC

Análise estatística: AF, AR Filho

Responsabilidade geral pelo estudo: $\mathrm{AF}$

Informações sobre financiamento: N/A

* Todos os autores leram e aprovaram a versão final submetida ao J Vasc Bras. 